\title{
Spectroscopy of the Central Stars of Three Old Planetary Nebulae
}

\author{
W. Saurer ${ }^{1}$, K. Werner ${ }^{2}$ and R. Weinberger ${ }^{1}$ \\ ${ }^{1}$ Institut für Astronomie, Universität Innsbruck; \\ ${ }^{2}$ Institut für Astronomie und Astrophysik, Universität Tübingen
}

The knowledge of the planetary nebula central star - white dwarf transition region has dramatically increased during the most recent years and dozens of stars now populate this former gap in the HRD. Each addition to the limited sample of central stars that has been studied spectroscopically in detail is, however, of value.

We present further results of a spectroscopic project that aims at investigations of stars in the upper left part of the HRD. Using EFOSC 1 at the ESO $3.6 \mathrm{~m}$ telescope in two nights in July 1995 we observed three central stars (CS) (PN G308.2+07.7, PN G011.4+17.9, and PN G332.5-16.9) of $15.2<\mathrm{V}<17.5 \mathrm{mag}$ with a dispersion of $39-73 \AA / \mathrm{mm}$ within 3600 $9000 \AA$. The data reduction was made using IRAF.

The spectra were analysed by fitting the $\mathrm{H}$ and $\mathrm{He}$ II lines with theoretical profiles from non-LTE model atmospheres. The models are composed of hydrogen and helium and disregard metal opacities which, however, yield reliable results. The influence of CNO as well as light metals and iron group elements on Balmer lines (high series members) and He II lines is almost negligible (e.g. Werner 1966, ApJ. 457, L39). Errors in the parameter determination are estimated to $10000 \mathrm{~K}$ in $\mathrm{T}_{\text {eff }}, 0.3$ dex in $\log \mathrm{g}$ and $\mathrm{He} / \mathrm{H}$ number ratio.NLTE models and line profiles were computed with the PRO 1 and LINE 1 codes which were mainly developed at the Kiel institute. Basic model assumptions are plane-parallel geometry, hydrostatic and radiative equilibrium.

Comparison with post-AGB tracks (e.g. Blöcker \& Schönberner 1990, A\&A 240, L11) reveals that the masses of the three CS range between 0.55 and $0.75 \mathrm{M}_{\odot}$. The stars appear to be hot (ca. $10^{5} \mathrm{~K}$ ), but normal CS. The central star of PN G332.5-16.9 is an exception, because it shows an enrichment in helium and probably also in carbon and nitrogen.

Acknowledgements: Thanks are due to the Austrian Ministry for Science, Transport and Arts for covering the travel expenses to Chile for R.W.. W.S. was supported financially by the "Jubiläumsfonds der Österreichischen Nationalbank", project 4713 (computer facilities). 